\title{
Distinctive signals of boosted dark matter from its semiannihilation
}

\author{
Takashi Toma $\odot^{*}$ \\ Institute of Liberal Arts and Science, Kanazawa University, Kanazawa 920-1192, Japan \\ and Institute for Theoretical Physics, Kanazawa University, Kanazawa 920-1192, Japan
}

(Received 13 September 2021; accepted 17 January 2022; published 10 February 2022)

\begin{abstract}
Dark matter can be boosted by various mechanisms, which may produce characteristic signals that are different from those of canonical dark matter. We show that the semiannihilation $\chi \chi \rightarrow \bar{\chi} \nu$ produces signals that are distinctive from those of other semiannihilation and standard dark matter annihilation processes. Because the boosted dark matter produced by the semiannihilation process is regarded as a high-energy neutrino, the total flux of the dark matter and the accompanying neutrino produce double peaks in the energy close to the dark matter mass. We show that it will be possible to detect both of the particles produced at the Sun using future large volume neutrino detectors such as those of the Deep Underground Neutrino Experiment and Hyper-Kamiokande.
\end{abstract}

DOI: 10.1103/PhysRevD.105.043007

\section{INTRODUCTION}

The nature of the dark matter in the universe is still unknown, and its investigation is a primary subject of astroparticle physics. Weakly interacting massive particles (WIMPs) are some of the well-motivated dark matter candidates. WIMPs were thermally produced in the early universe via sufficient interactions with standard model (SM) particles, and their relic abundance is determined by the so-called freeze-out mechanism without dependence on the initial conditions. However, no clear evidence for WIMPs has yet been found, and strong constraints are imposed on WIMP models. In particular, the current constraints on direct detection experiments are very severe. The XENON1T and PandaX-4T collaborations have set upper bounds on the WIMP-nucleon spin-independent (SI) elastic scattering cross section of $4.1 \times 10^{-47} \mathrm{~cm}^{2}$ and $3.3 \times 10^{-47} \mathrm{~cm}^{2}$ for a WIMP mass of $30 \mathrm{GeV}$, respectively $[1,2]$. One of the ideas for accommodating the strong constraints is to consider velocity-suppressed cross sections such as those of pseudo-Nambu-Goldstone boson dark matter [3] or fermion dark matter with a pseudoscalar mediator [4].

Another method is to consider semiannihilating dark matter, which is another kind of WIMP and emerges in models with a nonminimal dark sector. In general, semiannihilations are processes like $\chi \chi \rightarrow \bar{\chi} X$ with a (anti)dark

\footnotetext{
toma@staff.kanazawa-u.ac.jp
}

Published by the American Physical Society under the terms of the Creative Commons Attribution 4.0 International license. Further distribution of this work must maintain attribution to the author(s) and the published article's title, journal citation, and DOI. Funded by SCOAP ${ }^{3}$. matter particle, $\chi(\bar{\chi})$ and SM particle, $X$ in the final state $[5,6]{ }^{1}$ This kind of dark matter is also thermally produced via a freeze-out mechanism just like WIMPs. However, it can have relatively weaker couplings with the SM particles compared to WIMPs. For example, when an extra $U(1)$ symmetry is spontaneously broken to a $\mathbb{Z}_{3}$ symmetry, the lightest $\mathbb{Z}_{3}$ charged particle can be a candidate of semiannihilating dark matter. Semiannihilations possess some interesting features that are different from those of standard annihilations. First, the semiannihilating dark matter cannot be a self-conjugate particle in minimal models because of the charge conservation of the semiannihilating processes, as can be seen from $\chi \chi \rightarrow \bar{\chi} X$. Second, semiannihilations produce (semi)relativistic (anti)dark matter particles. Such boosted dark matter may be detectable using large volume neutrino detectors such as those of Super-Kamiokande (SK) [7], IceCube/DeepCore [8], and the next generation experiments at Hyper-Kamiokande (HK) [9], PINGU [10], the Deep Underground Neutrino Experiment (DUNE) [11], and $\mathrm{KN} 3 \mathrm{NeT}$ [12].

In this paper, we consider one of the most native semiannihilation processes, $\chi \chi \rightarrow \bar{\chi} \nu$ where $\nu$ is the light active neutrino in the SM, and investigate the simultaneous detection of both particles in the final state at large volume neutrino detectors such as those at SK/HK [9] and DUNE [11]. The similar signals produced by the boosted dark matter at the Galactic center and Sun were discussed in Refs. [13-15]. Furthermore, the boosted dark matter signals induced by excited dark matter and multicomponent dark matter scenarios [16-21], and alternative ideas for boosted dark matter detection [22,23] have also been studied.

\footnotetext{
${ }^{1}$ The corresponding $C P$ conjugate process, $\bar{\chi} \bar{\chi} \rightarrow \chi \bar{X}$ also exists.
} 


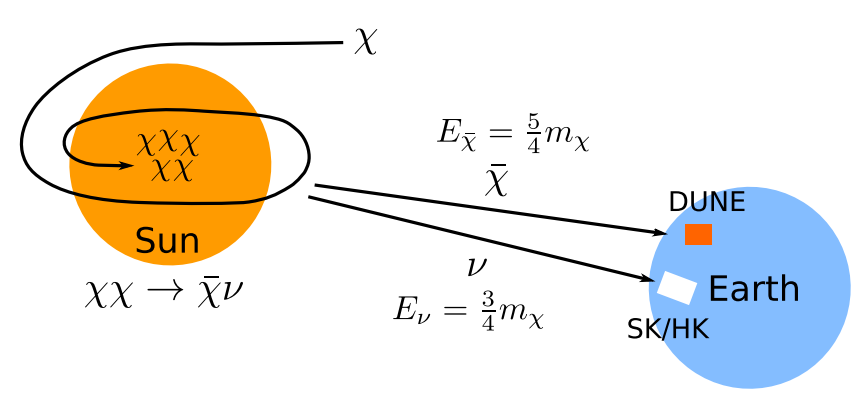

FIG. 1. Schematic of neutrino and boosted dark matter signals produced by dark matter semiannihilation at Sun. Both particles should be detectable at large volume neutrino detectors such as those at SK/HK and DUNE.

On the other hand, this paper focuses on a semiannihilation process that produces simultaneous signals from the boosted dark matter and high-energy neutrino, which are closely correlated with each other. This is different from the previous works, and has become a distinctive feature of the semiannihilation process. A schematic of these signals is depicted in Fig. 1.

\section{FLUXES FROM THE SUN}

We focus on the neutrino and boosted dark matter fluxes generated by the semiannihilation process $\chi \chi \rightarrow \bar{\chi} \nu$ in the Sun. Although it is also possible to consider the fluxes from the galactic center or dwarf spheroidal galaxies, the resultant $\bar{\chi}$ and $\nu$ fluxes are smaller than those from the Sun $[13,14]$. The energy fluxes of the neutrino and antidark matter from the Sun are given by the following: [24,25]

$$
\frac{d \Phi_{\nu / \bar{\chi}}}{d E_{\nu / \bar{\chi}}}=\frac{\Gamma_{\text {ann }}}{4 \pi d_{\odot}^{2}} \frac{d N_{\nu / \bar{\chi}}}{d E_{\nu / \bar{\chi}}},
$$

where $d_{\odot}=1.5 \times 10^{13} \mathrm{~cm}$ is the distance between the Sun and the Earth, $d N_{\nu / \bar{x}} / d E_{\nu / \bar{x}}$ are the energy spectra of $\nu$ and $\bar{\chi}$, and $\Gamma_{\text {ann }}$ is the annihilation rate, which is proportional to the semiannihilation cross section times the squared number of accumulated dark matter particles in the Sun: $\left\langle\sigma_{\chi \chi \rightarrow \bar{\chi}} v\right\rangle N_{\chi}^{2}$. The energies of $\nu$ and $\bar{\chi}$ are fixed at $E_{\nu}=$ $3 m_{\chi} / 4$ and $E_{\bar{\chi}}=5 m_{\chi} / 4$ respectively, because the annihilating dark matter particles in the initial state are nonrelativistic. Thus, the energy spectra $d N_{\nu / \bar{x}} / d E_{\nu / \bar{x}}$ are simply given by delta functions, and the energy difference between $\nu$ and $\bar{\chi}$ is exactly fixed at $E_{\bar{\chi}}-E_{\nu}=m_{\chi} / 2$. The total combined flux would exhibit a double peak structure, which can be a discriminative feature of the semiannihilation process. Note that the $C P$ conjugate process $\bar{\chi} \bar{\chi} \rightarrow \chi \bar{\nu}$ should also be taken into account if the observed relic abundance is occupied by both $\chi$ and $\bar{\chi}$.

The dark matter particles are captured in the center of the Sun as a result of the energy loss via elastic scattering with nucleons, while the number of accumulated antidark matter particles decrease via the semiannihilation. Here, we assume that the spin-dependent (SD) elastic scattering cross section $\sigma_{\mathrm{SD}}$ is dominant over the SI cross section and is velocity-dependent $\left(\sigma_{\mathrm{SD}} \propto v^{2}\right.$ with dark matter velocity $\left.v\right)$ because the SI cross section is strongly constrained by dark matter direct detection experiments [1,2]. This velocity dependence leads to significant enhancements of the boosted dark matter signals at the detectors because the typical dark matter local velocity is $v \sim 10^{-3}$ while it is $v=0.6$ for the boosted dark matter.

The evaporation effect of the accumulated dark matter particles in the Sun could be negligible if the dark matter mass is heavier than $4 \mathrm{GeV}$ [26]. Then, the capture and semiannihilation processes reach equilibrium after sufficient time, and the number of dark matter particles in the Sun becomes a constant. In this case, the annihilation rate in Eq. (1) can be written in terms of the capture rate, which is proportional to $\sigma_{\mathrm{SD}}[14,27]$. We use the analytic fitting function given by

$$
\Gamma_{\mathrm{ann}} \approx 2.6 \times 10^{21}\left[\mathrm{~s}^{-1}\right]\left(\frac{1 \mathrm{TeV}}{m_{\chi}}\right)^{C_{f}}\left(\frac{\sigma_{\mathrm{SD}}}{10^{-40} \mathrm{~cm}^{2}}\right),
$$

for the velocity-dependent SD cross section, where fitting coefficient $C_{f}=2.15+0.21 \log _{10}\left(m_{\chi} / \mathrm{TeV}\right)$, and the above fitting is valid in the mass range $1 \mathrm{GeV} \lesssim m_{\chi} \lesssim 1 \mathrm{TeV}$. Note that the rate is enhanced by a factor of approximately 20 compared to the constant $\sigma_{\mathrm{SD}}$ [14].

The SD elastic scattering cross section is constrained by the direct detection experiments. However it is not as strong as the SI cross section $[28,29]$. The PICO-60 experiment using $52 \mathrm{~kg}$ of $\mathrm{C}_{3} \mathrm{~F}_{8}$ set the strongest bound for $m_{\chi} \lesssim$ $100 \mathrm{GeV}$ [30], which was given by $3.4 \times 10^{-41} \mathrm{~cm}^{2}$ for a $30 \mathrm{GeV}$ WIMP mass. For a heavier dark matter mass, the strongest upper bound comes from the neutrino observation at IceCube [31]. The bound depends on the dark matter annihilation channels, and is especially strong for $\tau \bar{\tau}$ and $\nu \bar{\nu}$ channels. We adopt the $\nu \bar{\nu}$ bound from Ref. [31], with a factor of $1 / 2$ taking into account the fact that only one neutrino is produced at the semiannihilation. This is translated into the upper bound on the annihilation rate, $\Gamma_{\mathrm{ann}}$. Therefore, this implies that the flux of the neutrino and boosted dark matter is also bounded as follows:

$$
\Phi_{\nu / \bar{\chi}} \leq 9.2 \times 10^{-7}\left[\mathrm{~cm}^{-2} \mathrm{~s}^{-1}\right]\left(\frac{1 \mathrm{TeV}}{m_{\chi}}\right)^{C_{f}}\left(\frac{\sigma_{\mathrm{SD}}^{\exp }}{10^{-40} \mathrm{~cm}^{2}}\right),
$$

where $\sigma_{\mathrm{SD}}^{\mathrm{exp}}$ is the experimental upper bound of the SD cross section.

An example of the combined flux of $\nu$ and $\bar{\chi}$ is shown in Fig. 2, where the delta functions have been filtered with a Gaussian kernel, taking into account the detector energy resolution $[32,33]$. The total flux shows a double peak structure at $E_{\nu}$ and $E_{\bar{\chi}}$. Because atmospheric neutrinos can 


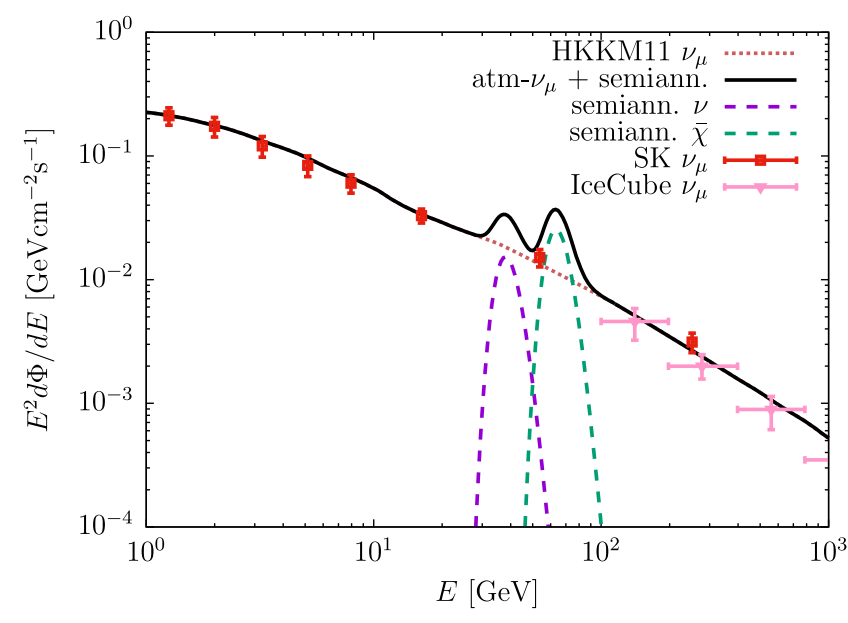

FIG. 2. Double peak structure of signal fluxes from Sun, where energy resolution of $\Delta E / E=25 \%$ is assumed for spectra. Values of $m_{\chi}=50 \mathrm{GeV}$ and $\sigma_{\mathrm{SD}}=3 \times 10^{-41} \mathrm{~cm}^{2}$ were selected for the dark matter mass and SD elastic scattering cross section, respectively, as an example. The red and pink points represent the experimental data of SK and IceCube, respectively [34]. The dark pink dotted line is the fitting with the HKKM11 model [35].

be the primary background for the signals, the total atmospheric muon neutrino fluxes observed at SK and IceCube are also shown in Fig. 2. Although atmospheric electron neutrinos also exist, the $\nu_{e}$ flux is much smaller than the muon neutrinos for the energy range $E_{\nu} \gtrsim \mathcal{O}(10) \mathrm{GeV}$.

\section{BOOSTED DARK MATTER SIGNAL AT DUNE}

DUNE consists of two detectors located at the Fermi National Accelerator Laboratory and Stanford Underground Research Laboratory [11]. The latter uses a 40 kton fiducial mass of liquid argon and can search for the signal from boosted dark matter scattering off a proton using a Liquid Argon Time Projection Chamber (LArTPC). ${ }^{2}$

The expected signal events of the boosted dark matter can be estimated by the following:

$$
N_{\text {sig }}=t_{\text {exp }} \cdot N_{p} \cdot \Phi_{\bar{\chi}} \cdot \tilde{\sigma}_{\mathrm{SD}} \cdot \epsilon_{\mathrm{eff}},
$$

where $t_{\exp }$ is the exposure time, $N_{p}$ is the number of target protons at the detector, $\Phi_{\bar{\chi}}$ is the flux from the Sun, $\tilde{\sigma}_{\mathrm{SD}}$ is the scattering cross section of the boosted dark matter with a proton in the argon detector, and $\epsilon_{\text {eff }}$ is the detector efficiency.

The elastic scattering cross section for the boosted dark matter $\left(\tilde{\sigma}_{\mathrm{SD}}\right)$ is different from $\sigma_{\mathrm{SD}}$ which appears in the above flux calculation. Because the SD cross section is

\footnotetext{
${ }^{2}$ Although scattering off a neutron can also produce a signal, the event reconstruction is relatively more challenging than that for a proton.
}

assumed to be velocity-suppressed $\left(\sigma_{\mathrm{SD}} \propto v^{2}\right)$ for nonrelativistic dark matter, the cross section $\tilde{\sigma}_{\mathrm{SD}}$ is approximately estimated as $\tilde{\sigma}_{\mathrm{SD}} \sim 3.6 \times 10^{5} \sigma_{\mathrm{SD}}$ taking into account the velocity difference between the nonrelativistic and boosted dark matter. Note that the effect of the nucleon form factors should also be taken into account for precise calculation, which is expected to induce a $\mathcal{O}(1)$ difference $[13,14,36]$.

From the kinematics of the elastic scattering, the energy of the scattered proton, $E_{p}$ is in the range of

$$
m_{p} \leq E_{p} \leq m_{p} \frac{\left(E_{\bar{\chi}}+m_{p}\right)^{2}+E_{\bar{\chi}}^{2}-m_{\chi}^{2}}{\left(E_{\bar{\chi}}+m_{p}\right)^{2}-E_{\bar{\chi}}^{2}+m_{\chi}^{2}}
$$

in the rest frame of the proton [13]. Substituting the energy of the boosted dark matter, $E_{\bar{\chi}}=5 m_{\chi} / 4$, the energy of the scattered proton becomes

$$
m_{p} \leq E_{p} \lesssim \frac{17}{8} m_{p}
$$

where $m_{p} \ll m_{\chi}$ is assumed. Thus, the proton energy is less than $2 \mathrm{GeV}$ and almost independent of the dark matter mass. In this energy range, resonant scatterings and deep inelastic scatterings can be ignored [36]. In addition, this energy is not sufficient to emit Cherenkov light in water and ice at facilities such as SK/HK and IceCube. However, it is anticipated to be observed at DUNE because the thresholds of the proton kinetic energy and angular resolution are as low as $50 \mathrm{MeV}$ and $5^{\circ}$, respectively [37]. These precise measurements significantly reduce the main background of atmospheric neutrinos [13].

The detector efficiency, $\epsilon_{\text {eff }}$ is estimated to be in the range of $0.80 \lesssim \epsilon_{\text {eff }} \lesssim 0.95$ for electron neutrinos and muon neutrinos via the charged current interaction when the neutrino energy is in the range of $1 \mathrm{GeV}$ to $2 \mathrm{GeV}$ [37]. Because there is no $\epsilon_{\text {eff }}$ estimation for the boosted dark matter, we selected $\epsilon_{\text {eff }}=0.80$ as a benchmark.

The expected number of signal events per year at DUNE is shown in Fig. 3. The number of target protons with the 40 kton fiducial mass of liquid argon is $N_{p}=1.1 \times 10^{34}$. The upper gray region is excluded by the bound of the SD cross section in the nonrelativistic limit. The left upper light-blue region is also excluded by the observation of the atmospheric neutrinos at SK. The SK limit is not as severe because it was evaluated with the atmospheric neutrinos from all directions. It is expected that the limit would become an order of magnitude more severe if only the direction of the Sun was taken into account. The red triangle is a sample parameter point that was adopted for Fig. 2. Note that a large number of signals can be expected if the dark matter mass is less than $\mathcal{O}(10) \mathrm{GeV}$. However, this mass region tends to be excluded by the gamma ray observations via the process $\chi \bar{\chi} \rightarrow q \bar{q}$ [38], which necessarily occurs through the crossing symmetry of the elastic 


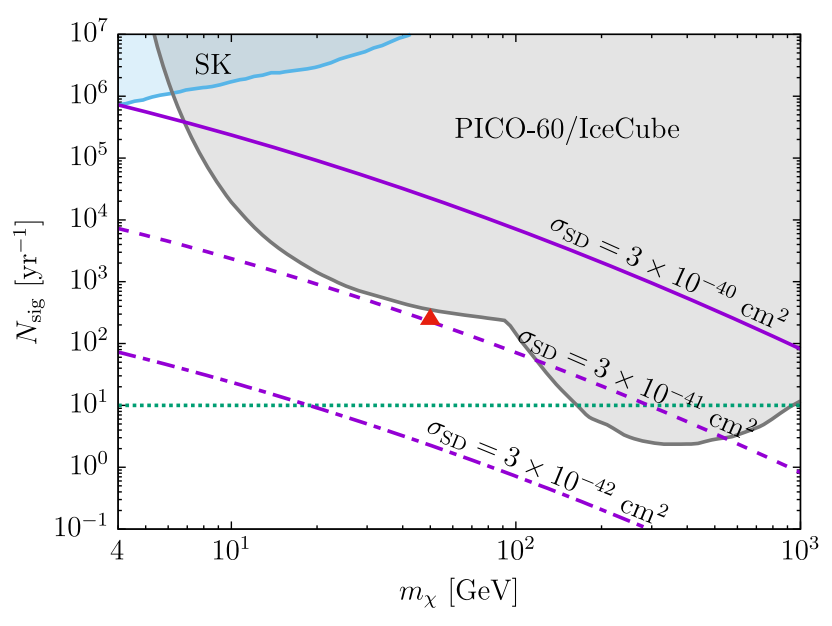

FIG. 3. Expected number of signal events at DUNE as function of dark matter mass, where detector efficiency is taken to be $\epsilon_{\text {eff }}=0.80$. The red triangle is a sample parameter point used for Fig. 2. The upper gray region is excluded by PICO-60 [30] and IceCube [31], while the left upper light-blue region is excluded by the observation of atmospheric neutrinos at SK [34].

scattering process, $\chi p \rightarrow \chi p$. The constraint depends on the dark matter models; it can be severe if the annihilation into quarks is $s$-wave, while there is no practical constraint for $p$-wave. For the realistic detection of a boosted dark matter signal, a benchmark for the number of events is set to be 10 events per year, which is shown as a green dotted line in Fig. 3. As seen in this figure, the dark matter mass should be $m_{\chi} \lesssim 150 \mathrm{GeV}$ so that the required number of events are observed. ${ }^{3}$ The expected number of signal events at the sample parameter point (red triangle) is 318 per year. The above calculation is no more than a rough estimation, and a more detailed simulation at DUNE should be performed following Refs. [11,36]. Furthermore, although we have not taken into account the effect of the neutrino oscillations, it should be included for a more sophisticated analysis.

In the case of a constant SD cross section, it is expected that the number of signal events would be smaller than that in the velocity-dependent case. Removing the enhancement factors for the $v^{2}$-dependent $\mathrm{SD}$ cross section, the number of signal events becomes 0.1 per year at most when the dark matter mass is $\sim 6 \mathrm{GeV}$ as can be seen in Fig. 3. Therefore, the velocity dependent cross section is essential for the signals of the boosted dark matter. Otherwise, a breakthrough in experimental technique is required.

The accompanying neutrino may also induce another signal, $\nu p \rightarrow \nu p$ at a different energy scale. Because

\footnotetext{
${ }^{3}$ Although a dark matter mass that is heavier than $\mathcal{O}(1) \mathrm{TeV}$ with a large SD cross section, may also produce the detectable number of signals, the accompanying neutrino would be outside of the energy threshold of DUNE. Even in this case, the neutrino could be detectable at other large volume neutrino detectors such as SK/HK [7,9], IceCube/DeepCore [8], PINGU [10], and KM3NeT [12].
}

$E_{\nu} \gg m_{p}$, deep inelastic scattering dominates in this case. The energy range of the scattered proton is given by $m_{p} \leq$ $E_{p} \lesssim m_{\chi}$ from Eq. (5). If an experiment at DUNE allows a neutrino signal search in this energy range, searches for the characteristic signals of both the boosted dark matter and neutrinos from the semiannihilation process can be conducted simultaneously, which is the best scenario. Even if this is not the case because the neutrino energy is too large (corresponding to a heavy dark matter mass) to be detected, a search for the signal from the accompanying neutrinos can be conducted at SK/HK $[7,9]$.

\section{MODEL BUILDING}

A velocity-dependent SD cross section was assumed in the above argument. Such a cross section can be derived from the anapole moment or scalar-pseudoscalar (SP) interaction given by the following: [39]

$$
\begin{aligned}
\mathcal{L}_{\text {ana }} & =\frac{1}{\Lambda^{2}} \bar{\chi} \gamma_{\mu} \gamma_{5} \partial_{\nu} \chi F^{\mu \nu}, \\
\mathcal{L}_{\mathrm{SP}} & =\frac{1}{\Lambda^{2}}(\bar{\chi} \chi)\left(\bar{q} \gamma_{5} q\right),
\end{aligned}
$$

where $\Lambda$ is the theoretical cutoff for the scale, and $F^{\mu \nu}$ is the electromagnetic field strength, which is contracted with the electromagnetic current $e A_{\mu} J^{\mu}$, and induces elastic scattering with a proton. The resultant SD cross sections from the above interactions are suppressed by the squared velocity $\left(\sigma_{\mathrm{SD}} \propto v^{2}\right)$ for nonrelativistic dark matter. However, note that for the anapole moment interaction, an SI cross section with the $v^{2}$ suppression also emerges at the same time, which could be dominant over the SD cross section.

In addition, the pseudoscalar-pseudoscalar (PP) interaction

$$
\mathcal{L}_{\mathrm{PP}}=\frac{1}{\Lambda^{2}}\left(\bar{\chi} \gamma_{5} \chi\right)\left(\bar{q} \gamma_{5} q\right),
$$

induces further suppression of the SD cross section $\left(\sigma_{\mathrm{SD}} \propto v^{4}\right)$. In this case, the standard annihilation cross section for $\chi \bar{\chi} \rightarrow q \bar{q}$ becomes $s$-wave, and the model is strongly constrained by the gamma ray observations of the MAGIC and Fermi-LAT collaborations if the dark matter mass is below $\mathcal{O}(100) \mathrm{GeV}$ [38].

When the velocity-suppression is extremely strong, like the above PP interaction, it is necessary to be careful with loop corrections in the ultra-violet (UV) complete models. The loop corrections may induce a new contribution to the cross section that is not velocity-suppressed, and becomes dominant over the velocity-suppressed cross sections, depending on the parameters.

In the UV complete models with radiative neutrino masses [40,41] and their further extensions [42], the semiannihilation process $\chi \chi \rightarrow \bar{\chi} \nu$ can naturally occur. 
In these models, although neutrino masses at the tree level are forbidden by the imposed $\mathbb{Z}_{3}$ symmetry, small masses are induced at the two-loop level. This symmetry also stabilizes the lightest $\mathbb{Z}_{3}$ charged fermion and provides a dark matter candidate with the semiannihilation process explored here.

\section{SUMMARY AND DISCUSSIONS}

We investigated the distinctive boosted dark matter and neutrino signals produced by the semiannihilation $\chi \chi \rightarrow \bar{\chi} \nu$ induced at the Sun. These two signals are correlated with each other, and are not produced by the other semiannihilation and standard dark matter annihilation processes. We approximately estimated the boosted dark matter and neutrino fluxes from the Sun, assuming a velocitysuppressed SD cross section. Both of the produced boosted dark matter and neutrino signals could be detected at DUNE and SK/HK. We also performed a simple estimation of the number of signal events at DUNE, and found that the dark matter mass should be $m_{\chi} \lesssim 150 \mathrm{GeV}$ to allow a realistic number of signal events to be observed. If these semiannihilation signals are experimentally confirmed, this will strongly suggest that the dark matter in the universe is a Dirac fermion with spin $1 / 2$. This is a unique process that makes it possible to identify the spin of the dark matter particle.

Our framework can be applied to other boosted dark matter scenarios such as multicomponent dark matter models. In this case, the correlation between the two signals would be weaker because the heavier dark matter captured in the Sun and the lighter boosted dark matter that could be detectable at DUNE are different particles. Furthermore, a similar argument with a velocity-dependent SI cross section could also be studied. In this case, the expected $\nu$ and $\bar{\chi}$ fluxes would be smaller than those in the case we have considered because the experimental limit on the SI cross section is much stronger than that of the SD cross section. However, at most, a few signal events per year from boosted dark matter can be expected at DUNE if the dark matter mass is less than $10 \mathrm{GeV}$.

\section{ACKNOWLEDGMENTS}

The author would like to thank Mayumi Aoki for carefully reading the manuscript and providing valuable comments. This work was supported by a JSPS Grant-in-Aid for Scientific Research (KAKENHI Grant No. JP20K22349). The numerical computation in this work was carried out at the Yukawa Institute Computer Facility.
[1] E. Aprile et al. (XENON Collaboration), Phys. Rev. Lett. 121, 111302 (2018).

[2] Y. Meng et al. (PandaX-4T Collaboration), Phys. Rev. Lett. 127, 261802 (2021).

[3] C. Gross, O. Lebedev, and T. Toma, Phys. Rev. Lett. 119, 191801 (2017).

[4] S. Ipek, D. McKeen, and A. E. Nelson, Phys. Rev. D 90, 055021 (2014).

[5] T. Hambye, J. High Energy Phys. 01 (2009) 028.

[6] F. D'Eramo and J. Thaler, J. High Energy Phys. 06 (2010) 109.

[7] C. Kachulis et al. (Super-Kamiokande Collaboration), Phys. Rev. Lett. 120, 221301 (2018).

[8] M. G. Aartsen et al. (IceCube Collaboration), Eur. Phys. J. C 75, 492 (2015).

[9] K. Abe et al. (Hyper-Kamiokande Collaboration), arXiv: 1805.04163.

[10] M. G. Aartsen et al. (IceCube-PINGU Collaboration), arXiv:1401.2046.

[11] B. Abi et al. (DUNE Collaboration), arXiv:2002.03005.

[12] S. Adrian-Martinez et al. (KM3Net Collaboration), J. Phys. G 43, 084001 (2016).

[13] K. Agashe, Y. Cui, L. Necib, and J. Thaler, J. Cosmol. Astropart. Phys. 10 (2014) 062.

[14] J. Berger, Y. Cui, and Y. Zhao, J. Cosmol. Astropart. Phys. 02 (2015) 005.

[15] D. McKeen and N. Raj, Phys. Rev. D 99, 103003 (2019).
[16] K. Kong, G. Mohlabeng, and J. C. Park, Phys. Lett. B 743, 256 (2015).

[17] J. Kopp, J. Liu, and X. P. Wang, J. High Energy Phys. 04 (2015) 105.

[18] H. Alhazmi, K. Kong, G. Mohlabeng, and J. C. Park, J. High Energy Phys. 04 (2017) 158.

[19] D. Kim, J. C. Park, and S. Shin, Phys. Rev. Lett. 119, 161801 (2017).

[20] M. Aoki and T. Toma, J. Cosmol. Astropart. Phys. 10 (2018) 020.

[21] D. Kim, J. C. Park, and S. Shin, Phys. Rev. D 100, 035033 (2019).

[22] D. Kim, K. Kong, J. C. Park, and S. Shin, J. High Energy Phys. 08 (2018) 155.

[23] D. McKeen, M. Pospelov, and N. Raj, Phys. Rev. Lett. 125, 231803 (2020).

[24] G. Belanger, F. Boudjema, A. Pukhov, and A. Semenov, Comput. Phys. Commun. 185, 960 (2014).

[25] P. Baratella, M. Cirelli, A. Hektor, J. Pata, M. Piibeleht, and A. Strumia, J. Cosmol. Astropart. Phys. 03 (2014) 053.

[26] G. Busoni, A. De Simone, and W. C. Huang, J. Cosmol. Astropart. Phys. 07 (2013) 010.

[27] R. Garani and S. Palomares-Ruiz, J. Cosmol. Astropart. Phys. 05 (2017) 007.

[28] E. Aprile et al. (XENON Collaboration), Phys. Rev. Lett. 122, 141301 (2019). 
[29] J. Xia et al. (PandaX-II Collaboration), Phys. Lett. B 792, 193 (2019).

[30] C. Amole et al. (PICO Collaboration), Phys. Rev. Lett. 118, 251301 (2017).

[31] M. G. Aartsen et al. (IceCube Collaboration), J. Cosmol. Astropart. Phys. 04 (2016) 022.

[32] G. Bertone, C. B. Jackson, G. Shaughnessy, T. M. P. Tait, and A. Vallinotto, Phys. Rev. D 80, 023512 (2009).

[33] A. Ibarra, S. Lopez Gehler, and M. Pato, J. Cosmol. Astropart. Phys. 07 (2012) 043.

[34] E. Richard et al. (Super-Kamiokande Collaboration), Phys. Rev. D 94, 052001 (2016).

[35] M. Honda, T. Kajita, K. Kasahara, and S. Midorikawa, Phys. Rev. D 83, 123001 (2011).
[36] J. Berger, Y. Cui, M. Graham, L. Necib, G. Petrillo, D. Stocks, Y. T. Tsai, and Y. Zhao, Phys. Rev. D 103, 095012 (2021).

[37] R. Acciarri et al. (DUNE Collaboration), arXiv:1512 .06148 .

[38] M. L. Ahnen et al. (MAGIC and Fermi-LAT Collaborations), J. Cosmol. Astropart. Phys. 02 (2016) 039.

[39] G. B. Gelmini, V. Takhistov, and S. J. Witte, J. Cosmol. Astropart. Phys. 07 (2018) 009; 02 (2019) E02.

[40] E. Ma, Phys. Lett. B 662, 49 (2008).

[41] M. Aoki and T. Toma, J. Cosmol. Astropart. Phys. 09 (2014) 016.

[42] S. Y. Ho, T. Toma, and K. Tsumura, Phys. Rev. D 94, 033007 (2016). 